\title{
Informed Consent Time
}

National Cancer Institute

\section{Source}

National Cancer Institute. Informed Consent Time. NCI Thesaurus. Code C162171.

The time of informed consent collection. 